\title{
New Genetic Insights from Autoimmune Thyroid Disease
}

\author{
Terry F. Davies, Rauf Latif, and Xiaoming Yin \\ Thyroid Research Unit, Mount Sinai School of Medicine, James J. Peters VA Medical Center, New York, NY 10468, USA \\ Correspondence should be addressed to Terry F. Davies, terry.davies@mssm.edu
}

Received 21 September 2011; Accepted 12 December 2011

Academic Editor: Juan Carlos Galofré

Copyright ( 2012 Terry F. Davies et al. This is an open access article distributed under the Creative Commons Attribution License, which permits unrestricted use, distribution, and reproduction in any medium, provided the original work is properly cited.

\begin{abstract}
The autoimmune thyroid diseases (AITDs) (Graves' disease and Hashimoto's thyroiditis) are complex genetic diseases which most likely have more than 20 genes contributing to the clinical phenotypes. To date, the genes known to be contributing fall into two categories: immune regulatory genes (including HLA, CTLA4, PTPN22, CD40, CD25, and FCRL3) and thyroid-specific genes (TG and TSHR). However, none of these genes contribute more than a 4-fold increase in risk of developing one of these diseases, and none of the polymorphisms discovered is essential for disease development. Hence, it appears that a variety of different gene interactions can combine to cause the same clinical disease pattern, but the contributing genes may differ from patient to patient and from population to population. Furthermore, this possible mechanism leaves open the powerful influence of the environment and epigenetic modifications of gene expression. For the clinician, this means that genetic profiling of such patients is unlikely to be fruitful in the near future.
\end{abstract}

\section{Introduction}

Many diseases have a tendency to run in families, and we know that this may be due to either environmental influences, or family genetics, or both. The autoimmune thyroid diseases (AITDs), Graves' disease and Hashimoto's thyroiditis, are typical examples of such complex diseases and have been recognized for many years as having an important genetic component. In the last 10 years we have learned many new insights into the way genetic influences can enhance thyroid autoimmunity, but there remain large gaps in our knowledge which are unlikely to be filled without major theoretical and technical advances. This brief review examines the current state of knowledge and what new insights we have gained from exploring the genetics of the AITDs, and in particular Graves' disease.

\section{Thyroid Autoantibodies}

Autoantibodies to thyroid peroxidase (TPO) and thyroglobulin $(\mathrm{Tg})$ are reflections of thyroid disease rather than causative agents [1]. Hence, such thyroid autoantibodies may develop before the onset of clinical AITD and have been long known to increase the risk of developing clinical AITD [2]. The recognition of a familial association for the production of thyroid antibodies [3] led to studies of firstdegree relatives of probands with AITD and indicated a dominant pattern of inheritance. Indeed, up to $50 \%$ of the siblings of patients with AITD are thyroid antibody positive $[4,5]$ in contrast to $\sim 15 \%$ in the general population [6]. Several segregation analyses have also shown a Mendelian dominant pattern of inheritance for the expression of thyroid autoantibodies $[7,8]$, and genetic transmission of TPO antibody subclass "fingerprints" has suggested that the pattern of autoantibody recognition of the TPO antigen was also genetically transmitted [9].

\section{Genetic Susceptibility to AITD}

The recognition of an association between AITD and certain human leukocyte antigens (HLA) first provided a mechanism for the genetic contribution to Graves' disease and Hashimoto's thyroiditis [10]. This association has been especially well seen in identical twins [11]. The HLA antigens provide a means for the immune system to recognize thyroid antigenic peptides, and recent data have demonstrated this enhanced association as secondary to the presence of particular residues in the HLA class II binding pocket such as Arg 74 [12]. In addition, as the pathological and molecular 
TABLE 1: Methods of genetic analysis.

(A) Linkage analysis

This is based on the principle that the chance for a recombination event between 2 loci (i.e., a marker, such as the candidate gene, and the true disease gene) is proportional to the chromosomal distance between them. Therefore, if a marker is close to a disease susceptibility gene, this marker will cosegregate with the disease in families.

The logarithm of odds (LOD) score is a measure of the evidence for or against linkage between a marker and a trait or disease [13]. LOD score analysis has had important advantages for the study of AITD because it has allowed a way to test for the presence of heterogeneity within the data set and allowed deduction of the mode of inheritance and the degree of penetrance from the linkage data.

Linkage studies are highly specific but have been clearly shown not to be highly sensitive.

\section{(B) Association studies}

These studies simply compare the presence of a disease marker (such as the candidate gene) in the disease population with the presence of the marker in a control population without the disease.

Here, the difficulty may lie in the appropriate control population, which needs to be comparable and large.

If this difficulty is overcome, association studies can reveal a genetic influence, and with large patient groups, this type of study can be highly sensitive.

mechanisms involved in AITD became known, many of which were not only common to all autoimmune diseases but also highly variable between individuals; this allowed the recognition of candidate genes responsible for disease susceptibility. Such genes could then be assessed by either linkage analysis or association studies (see Table 1).

\section{Detecting Susceptibility Genes in AITD}

The candidate HLA gene complex was first associated with AITD in association studies but then failed to show linkage with AITD [14]. This showed that the genetic contribution of HLA to AITD was not strong enough to be seen in linkage analyses [13]. This indicated that association studies were more likely to detect genes contributing small effects on disease susceptibility. As a consequence of the Human Genome Project, it became possible to identify genes for diseases that had a complex genetic basis without resorting to the candidate gene approach. This was achieved by "typing" individuals using a genome screen of genetic markers, at first with microsatellites ( 1 microsatellite per $10 \mathrm{cM}$ DNA) and later single-nucleotide polymorphisms (SNPs) ( 1 SNP per $<1$ cM DNA), which covered the entire genome (Table 2) [15]. Then investigators observed which markers segregated with the disease. However, the reduced sensitivity of linkage analyses, compared to association studies, made it more difficult to perform these analyses for the complex traits characteristic of a non-Mendelian pattern of inheritance and with variable clinical phenotypes. However, using large
TABLE 2: Methods for whole-genome screening.

(A) Microsatellites

These are regions in the genome that are composed of repetitive sequences. The most common microsatellites are the CA (dC-dA)n repeats. Microsatellite loci are highly polymorphic because of variation in the number of repeats (usually there are 5 to 15 alleles per locus), and they are uniformly distributed throughout the genome at distances of fewer than 1 million base pairs [15]. Therefore, microsatellites served as useful markers in linkage studies designed to search for unknown disease susceptibility genes. Investigators then further narrowed the suspected gene region with more dense markers, and the gene could be identified.

(B) Single-nucleotide polymorphisms (SNPs)

Without having to enlist families, it is now possible to use genome-wide association studies involving up to $10^{6} \mathrm{SNPs}$ (on a microchip), each of which is in linkage disequilibrium with large segments of the genome, and then analyze their association with any disease.

numbers of SNPs, developed as a result of the HapMap project $[16,17]$, and which had a much greater degree of coverage of the whole genome, it was easier to decipher which markers segregated with the disease using association analyses. These SNP markers occur more frequently than microsatellite markers and are easy to detect, allowing for greater genetic sensitivity. The suspected gene region can then be further narrowed with more dense SNPs and the gene can be identified. Results are now available for a variety of autoimmune diseases including rheumatoid arthritis and type 1 diabetes mellitus [18] and most recently for AITD [19].

It is obviously essential that whole-genome association study results must be reliably and repeatedly reproduced, but the complexity of this type of analysis and the high cost have raised problems [20, 21]. If common diseases are associated with common risks, then replication across populations can be expected. But common diseases may be related to population-specific risks, and, therefore, such data can only be reproduced in the same population as that which was studied in the original report. Reproducibility had been a problem for studies that used microsatellite screening, including the studies in patients with AITD, and this problem has persisted in the much larger studies using whole-genome association studies such as in those analyzing Parkinson's disease and also obesity. Hence, all reports of genetic linkage and association require confirmation by independent studies before they can be accepted.

\section{Genes for AITD}

The HLA and CTLA4 genes were the first genes identified by the candidate approach $[22,23]$ (Table 3 ).

As discussed earlier, the HLA genes make up the major histocompatibility complex (MHC) which contains many genes related to immune system function in humans. These include HLA class I (A, B, and C), HLA class II (DP, DM, DOA, DOB, DQ, and DR), and HLA class III (coding for 
TABLE 3: Genes linked and/or associated with autoimmune thyroid disease.

\begin{tabular}{llcc}
\hline Gene symbol & Gene name & Chromosome location & Odds ratio \\
\hline HLA & Major histocompatibility complex & $6 \mathrm{p} 21$ & $2.0-4.0$ \\
CTLA4 & Cytotoxic T-lymphocyte-associated protein 4 & $2 \mathrm{q} 33$ & $1.5-2.2$ \\
PTPN22 & Protein tyrosine phosphatase, non-receptor type 22 (lymphoid) & $1 \mathrm{p} 13$ & $1.4-1.9$ \\
CD40 & CD40 molecule, TNF receptor superfamily member 5 & $20 \mathrm{q} 11$ & $1.3-1.8$ \\
IL2RA (CD25) & Interleukin 2 receptor, alpha & $10 \mathrm{p} 15$ & $1.1-1.4$ \\
FCRL3 & Fc receptor-like 3 & $1 \mathrm{q} 23$ & $1.1-1.3$ \\
TG & Thyroglobulin & $8 \mathrm{q} 24$ & $1.3-1.6$ \\
TSHR & Thyroid-stimulating hormone receptor & $14 \mathrm{q} 31$ & $1.4-2.6$ \\
\hline
\end{tabular}

other immune proteins). The major GD-associated $H L A$, $H L A-D R 3$, locates at the HLA DR locus and plays a key role in the normal immune response by binding peptide antigens and presenting them to T-cell receptors.

The cytotoxic T-lymphocyte-associated protein 4 (CTL A4) gene is an immune regulatory molecule, which is expressed on the surface of Helper T cells and transmits an inhibitory signal to T cells. In addition to the HLA and CTLA4 gene loci, there are confirmed associations (2 or more reports) for a number of genes also common to many autoimmune diseases: PTPN22, CD40, IL2RA (CD25), and FCRL3 (Table 3).

The gene for protein tyrosine phosphatase, non-receptor type 22 (lymphoid), also known as just PTPN22, encodes a protein tyrosine phosphatase expressed primarily in lymphoid tissues. This enzyme associates with the molecular adapter protein $\mathrm{CBL}$ and may be involved in regulating $\mathrm{CBL}$ function in the T-cell receptor signaling pathway. A variant of the PTPN22 encodes Lyp phosphatase (Lyp620W) and confers risk for multiple autoimmune diseases. Most recently, Zhang et al. [24] reported that levels of the Lyp620W variant were decreased in human $\mathrm{T}$ and $\mathrm{B}$ cells, and its calpain binding and cleavage were increased relative to wild-type Lyp620R. Therefore, calpain-mediated degradation with consequently reduced Lyp expression and lymphocyte and dendritic cell hyperresponsiveness represents a potential mechanism for unregulated autoimmunity. The LypR620W variant, with an arginine to tryptophan substitution, loses its function and influence on immune responses, which increases the risk for autoimmune disease.

The CD40 molecule, or TNF receptor superfamily member 5 gene, encodes a costimulatory receptor which is essential in mediating a broad variety of immune and inflammatory responses including T-cell-dependent immunoglobulin class switching, memory B-cell development, and germinal center formation [25]. The interleukin 2 (IL2) receptor alpha gene (IL2RA or CD25) encodes one of the subunits of the IL2 receptor that binds IL-2 and is vital in the regulation of Tcell function. The Fc receptor-like protein 3 (FCRL3) gene encodes a protein containing an immunoreceptor-tyrosine activation motif and immunoreceptor-tyrosine inhibitory motif in its cytoplasmic domain and may play a role in immune regulation.

To date, the only thyroid-related genes associated with AITD are TG (the gene encoding thyroglobulin) [26], in both
Graves' disease and Hashimoto's thyroiditis, and TSHR (the gene encoding the thyrotropin receptor) restricted to Graves' disease [27, 28] (Table 3).

The thyroglobulin $(T G)$ gene encodes a large glycoprotein homodimer produced exclusively by the thyroid gland. It acts as a substrate for the synthesis of thyroid hormones thyroxine (T4) and triiodothyronine (T3) as well as the storage of the inactive forms of thyroid hormone and iodine. How this gene influences susceptibility is unclear but Stefan et al. [29] have recently described a genetic/epigenetic mechanism by which a newly identified $T G$ promoter SNP variant predisposes to AITD. Sequencing analyses followed by case control and family-based association studies identified a SNP $(-1623 \mathrm{~A} \rightarrow \mathrm{G})$ that was associated with AITD in the Caucasian population, and the associated nucleotide substitution SNP $(-1623 \mathrm{~A} / \mathrm{G})$ modified a binding site for interferon regulatory factor-1 (IRF-1), a major interferoninduced transcription factor, indicating enhanced sensitivity to this inflammatory cytokine [29].

The thyroid stimulating hormone receptor (TSHR) gene encodes a membrane protein that signals through binding TSH ligand and is a major controller of thyroid cell growth and metabolism. SNPs in intron 1 (in Caucasians) and intron 7 (in Japanese) have been associated with Graves' disease in a number of studies $[27,28,30]$. Recent data suggest that TSHR-associated SNPs are related to defective thymic tolerance for the TSHR as shown by reduced expression within the thymus gland where it is needed to delete TSHR autoreactive T cells [31].

Because all the identified susceptibility genes found to date appear to have a low level of contribution to genetic susceptibility, a number of whole-genome screening studies have also been attempted in AITD to find more important genes [32-36]. One whole-genome association study using only $10^{4}$ nonsynonymous SNPs (those involving parts of a gene likely to affect the product character) showed a number of the previously recognized genes, as well as locating some new sites, but the new sites could not subsequently be confirmed [37, 38]. Most recently, the first full genomewide study of Graves' disease with $660 \mathrm{~K}$ SNPs has now been reported from China [19]. This study again identified many of the known genes for AITD, but also described two new sites on chromosomes $6 \mathrm{q}$ and $4 \mathrm{p}$. These await further confirmation. Again, however, no very highly associated new genes have emerged. 


\section{The Degree of Enhanced Susceptibility Remains Low}

All the genes associated with AITD are individually able to confer only modest degrees of disease susceptibility (expressed as odds ratios, see Table 3). Hence, these data only allow us to conclude that the AITDs, both Graves' disease (including Graves' ophthalmopathy) and Hashimoto's thyroiditis, are complex genetic disorders involving multiple genes that may interact to provide a susceptible background for disease development. Furthermore, there appear to be disease-specific genes, such as the gene encoding the TSHR in Graves' disease and a larger group of susceptibility genes, such as CTLA4, which are common to many autoimmune diseases. This combination of gene polymorphisms likely allows epigenetic phenomena, subsequent to a variety of influences such as infection and the environment, to initiate disease.

\section{The Controversy over Major Genes in AITD}

After the clarification that multiple genes are at work in AITD, it is likely that more than 20 potential genes contribute to the AITD phenotypes. But major genes, those essential to disease development, have not been found [39]. A major gene should be involved in the majority of patients with the disease, and the risk ratios, even for HLA, do not reveal such a gene (Table 3 ). This most likely means that different combinations of genes may produce similar clinical phenotypes or that epigenetic phenomena are dominant. So far, in the whole-genome screening of families, siblings, and populations with AITD, a number of sites have been established for Graves' disease and Hashimoto's thyroiditis susceptibility, but none of them have had very high statistical values (LOD scores) $[32,33,35]$. This finding has been true not just for AITD, but also for other autoimmune diseases including type 1 diabetes mellitus. This is best understood by thinking of HLA once again. Not every patient with Graves' disease has the associated HLA-DR3 subtype and not even the associated Arg74 in its binding pocket, irrespective of the HLA-DR subtype [12]. Hence, the disease can occur in the absence of the expected HLA association.

\section{A Note on Epigenetics}

One mechanism by which environmental factors may combine with genetic risk to promote AITD is by altering the epigenetic control of gene expression as seen, for example, in the pancreas [40] and as shown for a virus interacting with a susceptibility gene in Crohn's disease [41]. While little is known about such interactions with AITD, there has been wide confirmation of a role for $\mathrm{X}$ chromosome inactivation (XCI) $[42,43]$. Patients with AITD more often than expected showed a biased expression of a maternal or paternal $\mathrm{X}$ chromosome leading to the hypothesis that the poorly expressed chromosome could become active in certain tissues such as the thyroid and express new antigenic sequences not previously recognized by the immune system.
These potential mechanisms for enhanced susceptibility to AITD require further exploration.

\section{Conclusions}

How environmental factors combine with genetic risk at the molecular level to promote complex genetic diseases such as AITD is largely unknown. The genes that are linked to and/or associated with AITD are each small contributors to genetic risk. Multiple-gene polymorphisms (combinations of haplotypes) appear to be needed to develop AITD and may differ between geographic populations secondary to epigenetic influences. Much remains to be learned.

\section{Abbreviations}

AITD: Autoimmune thyroid disease

LOD: Logarithm of odds

SNP: Single-nucleotide polymorphism.

\section{Disclosure}

TFD is a Board Member of Kronus Inc., Star, Idaho (a distributor of thyroid antibody test kits). The other authors have no conflict of interests to disclose.

\section{Acknowledgments}

This work is supported in part by DK052464 and DK069713 from the National Institute of Diabetes and Digestive and Kidney Diseases, the VA Merit Award program, and the David Owen Segal Endowment.

\section{References}

[1] T. Davies, "Hypothyroidism and thyroiditis," in Williams Textbook of Endocrinology, J. Wilson, D. W. Foster, P. R. Larsen, and H. Kronenberg, Eds., pp. 423-456, W.B. Saunders, Orlando, Fla, USA, 2002.

[2] M. P. J. Vanderpump, W. M. G. Tunbridge, J. M. French et al., "The incidence of thyroid disorders in the community: a twenty-year follow-up of the Whickham Survey," Clinical Endocrinology, vol. 43, no. 1, pp. 55-68, 1995.

[3] R. Hall and J. B. Stanbury, "Familial studies of autoimmune thyroiditis," Clinical and Experimental Immunology, vol. 2, pp. 719-725, 1967.

[4] I. J. Chopra, D. H. Solomon, and U. Chopra, "Abnormalities in thyroid function in relatives of patients with Graves' disease and Hashimoto's thyroiditis: lack of correlation with inheritance of HLA B8," Journal of Clinical Endocrinology and Metabolism, vol. 45, no. 1, pp. 45-54, 1977.

[5] C. L. Burek, W. H. Hoffman, and N. R. Rose, "The presence of thyroid autoantibodies in children and adolescents with autoimmune thyroid disease and in their siblings and parents," Clinical Immunology and Immunopathology, vol. 25, no. 3, pp. 395-404, 1982.

[6] W. M. G. Tunbridge, D. C. Evered, and R. Hall, "The spectrum of thyroid disease in a community: the Whickham survey," Clinical Endocrinology, vol. 7, no. 6, pp. 481-493, 1977. 
[7] D. L. Pauls, M. Zakarija, J. M. McKenzie, and J. A. Egeland, "Complex segregation analysis of antibodies to thyroid peroxidase in Old Order Amish families," American Journal of Medical Genetics, vol. 47, no. 3, pp. 375-379, 1993.

[8] D. Phillips, L. Prentice, M. Upadhyaya et al., "Autosomal dominant inheritance of autoantibodies to thyroid peroxidase and thyroglobulin-studies in families not selected for autoimmune thyroid disease," Journal of Clinical Endocrinology and Metabolism, vol. 72, no. 5, pp. 973-975, 1991.

[9] J. C. Jaume, J. Guo, D. L. Pauls et al., "Evidence for genetic transmission of thyroid peroxidase autoantibody epitopic 'fingerprints," Journal of Clinical Endocrinology and Metabolism, vol. 84, no. 4, pp. 1424-1431, 1999.

[10] N. R. Farid and J. C. Bear, "The human major histocompatibility complex and endocrine disease," Endocrine Reviews, vol. 2, no. 1, pp. 50-86, 1981.

[11] T. H. Brix, K. Christensen, N. V. Holm, B. Harvald, and L. Hegedüs, "A population-based study of graves' disease in danish twins," Clinical Endocrinology, vol. 48, no. 4, pp. 397400, 1998.

[12] Y. Ban, T. F. Davies, D. A. Greenberg et al., "Arginine at position 74 of the HLA-DR $\beta 1$ chain is associated with Graves' disease," Genes and Immunity, vol. 5, no. 3, pp. 203-208, 2004.

[13] J. Ott, "Complex traits on the map," Nature, vol. 379, no. 6568, pp. 772-773, 1996.

[14] S. H. Roman, D. Greenberg, P. Rubinstein, S. Wallenstein, and T. F. Davies, "Genetics of autoimmune thyroid disease: lack of evidence for linkage to HLA within families," Journal of Clinical Endocrinology and Metabolism, vol. 74, no. 3, pp. 496503, 1992.

[15] J. L. Weber, "Human DNA polymorphisms and methods of analysis," Current Opinion in Biotechnology, vol. 1, no. 2, pp. 166-171, 1990.

[16] K. A. Frazer, D. G. Ballinger, D. R. Cox et al., "A second generation human haplotype map of over 3.1 million SNPs," Nature, vol. 449, no. 7164, pp. 851-861, 2007.

[17] K. Christensen and J. C. Murray, "Focus on research: what genome-wide association studies can do for medicine," New England Journal of Medicine, vol. 356, no. 11, pp. 1094-1097, 2007.

[18] T. A. Manolio, L. D. Brooks, and F. S. Collins, "A HapMap harvest of insights into the genetics of common disease," Journal of Clinical Investigation, vol. 118, no. 5, pp. 1590-1605, 2008.

[19] X. Chu, C.-M. Pan, S.-X. Zhao et al., "A genome-wide association study identifies two new risk loci for Graves' disease," Nature Genetics, vol. 43, no. 9, pp. 897-901, 2011.

[20] D. J. Hunter and P. Kraft, "Drinking from the fire hosestatistical issues in genomewide association studies," New England Journal of Medicine, vol. 357, no. 5, pp. 436-439, 2007.

[21] D. Shriner, L. K. Vaughan, M. A. Padilla, and H. K. Tiwari, "Problems with genome-wide association studies," Science, vol. 316, no. 5833, pp. 1840-1841, 2007.

[22] T. Yanagawa, Y. Hidaka, V. Guimaraes, M. Soliman, and L. J. DeGroot, "CTLA-4 gene polymorphism associated with Graves' disease in a Caucasian population," Journal of Clinical Endocrinology and Metabolism, vol. 80, no. 1, pp. 41-45, 1995.

[23] N. R. Farid, "Immunogenetics of autoimmune thyroid disorders," Endocrinology and Metabolism Clinics of North America, vol. 16, no. 2, pp. 229-245, 1987.

[24] J. Zhang, N. Zahir, Q. Jiang et al., "The autoimmune diseaseassociated PTPN22 variant promotes calpain-mediated Lyp/ Pep degradation associated with lymphocyte and dendritic cell hyperresponsiveness," Nature Genetics, vol. 43, no. 9, pp. 902$907,2011$.

[25] Y. Tomer, "Genetic susceptibility to autoimmune thyroid disease: past, present, and future," Thyroid, vol. 20, no. 7, pp. 715$725,2010$.

[26] Y. Tomer, D. A. Greenberg, E. Concepcion, Y. Ban, and T. F. Davies, "Thyroglobulin is a thyroid specific gene for the familial autoimmune thyroid diseases," Journal of Clinical Endocrinology and Metabolism, vol. 87, no. 1, pp. 404-407, 2002.

[27] X. Yin, R. Latif, R. Bahn, Y. Tomer, and T. F. Davies, "Influence of the TSH receptor gene on susceptibility to Graves' disease and Graves' ophthalmopathy," Thyroid, vol. 18, no. 11, pp. 1201-1206, 2008.

[28] B. M. Dechairo, D. Zabaneh, J. Collins et al., "Association of the TSHR gene with Graves' disease: the first disease specific locus," European Journal of Human Genetics, vol. 13, no. 11, pp. 1223-1230, 2005.

[29] M. Stefan, E. M. Jacobson, A. K. Huber et al., "Novel variant of thyroglobulin promoter triggers thyroid autoimmunity through an epigenetic interferon $\alpha$-modulated mechanism," Journal of Biological Chemistry, vol. 286, no. 36, pp. 3116831179, 2011.

[30] H. Hiratani, D. W. Bowden, S. Ikegami et al., "Multiple SNPs in intron 7 of thyrotropin receptor are associated with Graves' disease," Journal of Clinical Endocrinology and Metabolism, vol. 90, no. 5, pp. 2898-2903, 2005.

[31] R. Colobran, M. d. P. Armengol, R. Faner et al., "Association of an SNP with intrathymic transcription of TSHR and graves' disease: a role for defective thymic tolerance," Human Molecular Genetics, vol. 20, no. 17, pp. 3415-3423, 2011.

[32] Y. Tomer, G. Barbesino, D. A. Greenberg, E. Concepcion, and T. F. Davies, "Mapping the major susceptibility loci for familial Graves' and Hashimoto's diseases: evidence for genetic heterogeneity and gene interactions," Journal of Clinical Endocrinology and Metabolism, vol. 84, no. 12, pp. 4656-4664, 1999.

[33] Y. Tomer, Y. Ban, E. Conception et al., "Common and unique susceptibility loci in Graves and Hashimoto diseases: results of whole-genome screening in a data set of 102 multiplex families," American Journal of Human Genetics, vol. 73, no. 4, pp. 736-747, 2003.

[34] Y. Tomer, F. Menconi, T. F. Davies et al., "Dissecting genetic heterogeneity in autoimmune thyroid diseases by subset analysis," Journal of Autoimmunity, vol. 29, no. 2-3, pp. 69-77, 2007.

[35] J. C. Taylor, S. C. Gough, P. J. Hunt et al., "A genomewide screen in 1119 relative pairs with autoimmune thyroid disease," Journal of Clinical Endocrinology and Metabolism, vol. 91, no. 2, pp. 646-653, 2006.

[36] K. Sakai, S. Shirasawa, N. Ishikawa et al., "Identification of susceptibility loci for autoimmune thyroid disease to $5 \mathrm{q} 31$ q33 and Hashimoto's thyroiditis to 8q23-q24 by multipoint affected sib-pair linkage analysis in Japanese," Human Molecular Genetics, vol. 10, no. 13, pp. 1379-1386, 2001.

[37] P. R. Burton, D. G. Clayton, L. R. Cardon et al., "Association scan of 14,500 nonsynonymous SNPs in four diseases identifies autoimmunity variants," Nature Genetics, vol. 39, no. 11, pp. 1329-1337, 2007.

[38] P. R. Newby, O. J. Pickles, S. Mazumdar et al., "Follow-up of potential novel graves' disease susceptibility loci, identified in the UK WTCCC genome-wide nonsynonymous SNP study," 
European Journal of Human Genetics, vol. 18, no. 9, pp. 10211026, 2010.

[39] T. F. Davies, "Really significant genes for autoimmune thyroid disease do not exist—-so how can we predict disease?" Thyroid, vol. 17, no. 11, pp. 1027-1029, 2007.

[40] I. Sandovici, N. H. Smith, M. D. Nitert et al., "Maternal diet and aging alter the epigenetic control of a promoter-enhancer interaction at the Hnf4a gene in rat pancreatic islets," Proceedings of the National Academy of Sciences of the United States of America, vol. 108, no. 13, pp. 5449-5454, 2011.

[41] K. Cadwell, K. K. Patel, N. S. Maloney et al., "Virus-plussusceptibility gene interaction determines Crohn's disease gene Atg16L1 phenotypes in intestine," Cell, vol. 141, no. 7, pp. 1135-1145, 2010.

[42] T. H. Brix, G. P. S. Knudsen, M. Kristiansen, K. O. Kyvik, K. H. Ørstavik, and L. Hegedüs, "High frequency of skewed $\mathrm{x}$-chromosome inactivation in females with autoimmune thyroid disease: a possible explanation for the female predisposition to thyroid autoimmunity," Journal of Clinical Endocrinology and Metabolism, vol. 90, no. 11, pp. 5949-5953, 2005.

[43] X. Yin, R. Latif, Y. Tomer, and T. F. Davies, "Thyroid epigenetics: $\mathrm{X}$ chromosome inactivation in patients with autoimmune thyroid disease," Annals of the New York Academy of Sciences, vol. 1110, pp. 193-200, 2007. 


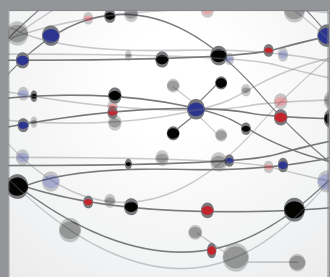

The Scientific World Journal
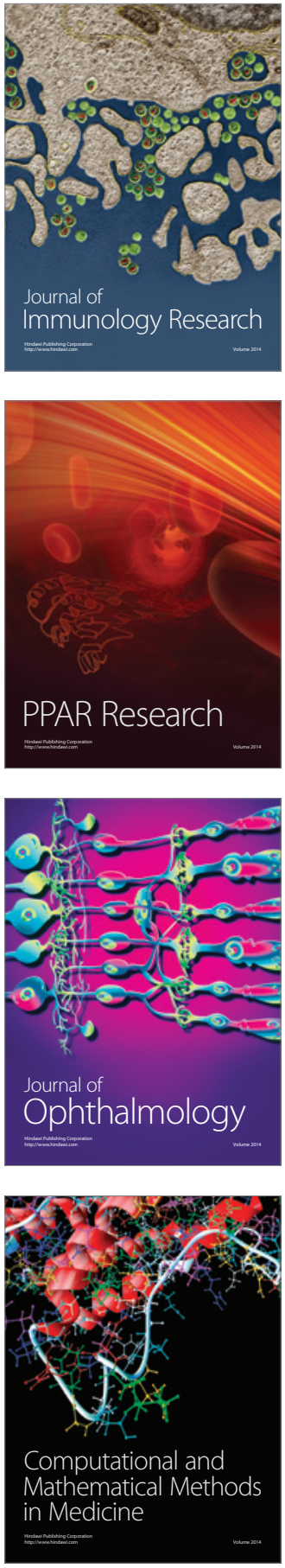

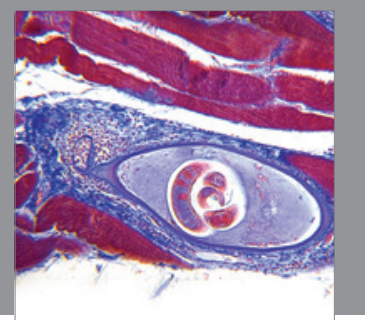

Gastroenterology

Research and Practice
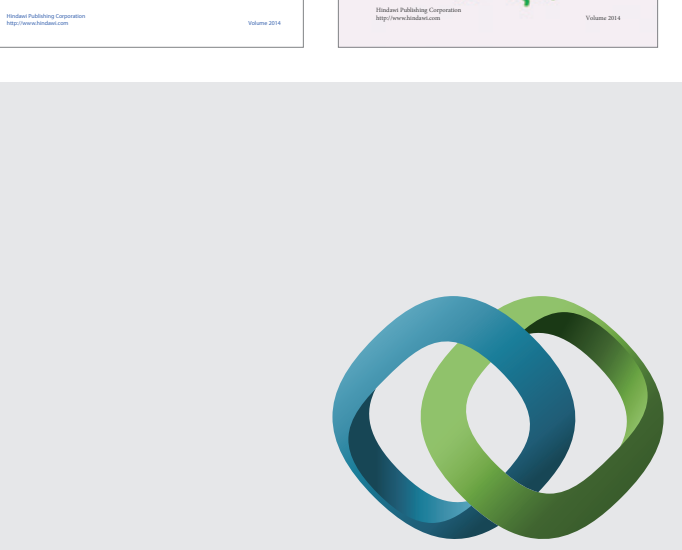

\section{Hindawi}

Submit your manuscripts at

http://www.hindawi.com
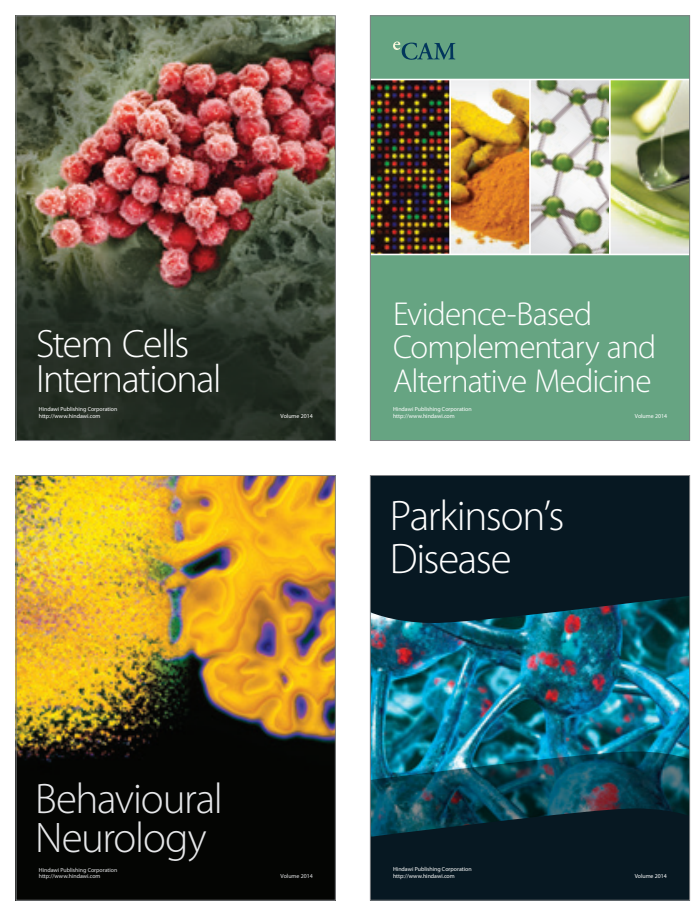

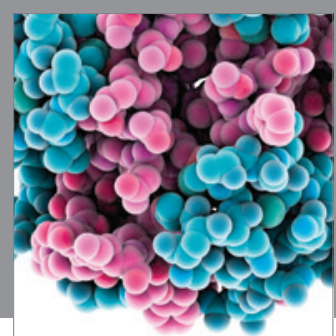

Journal of
Diabetes Research

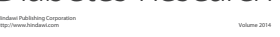

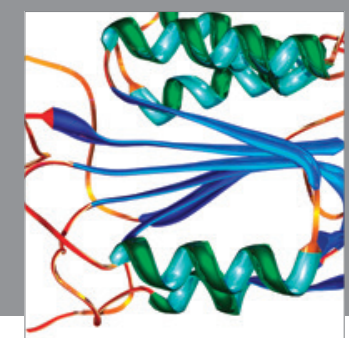

Disease Markers
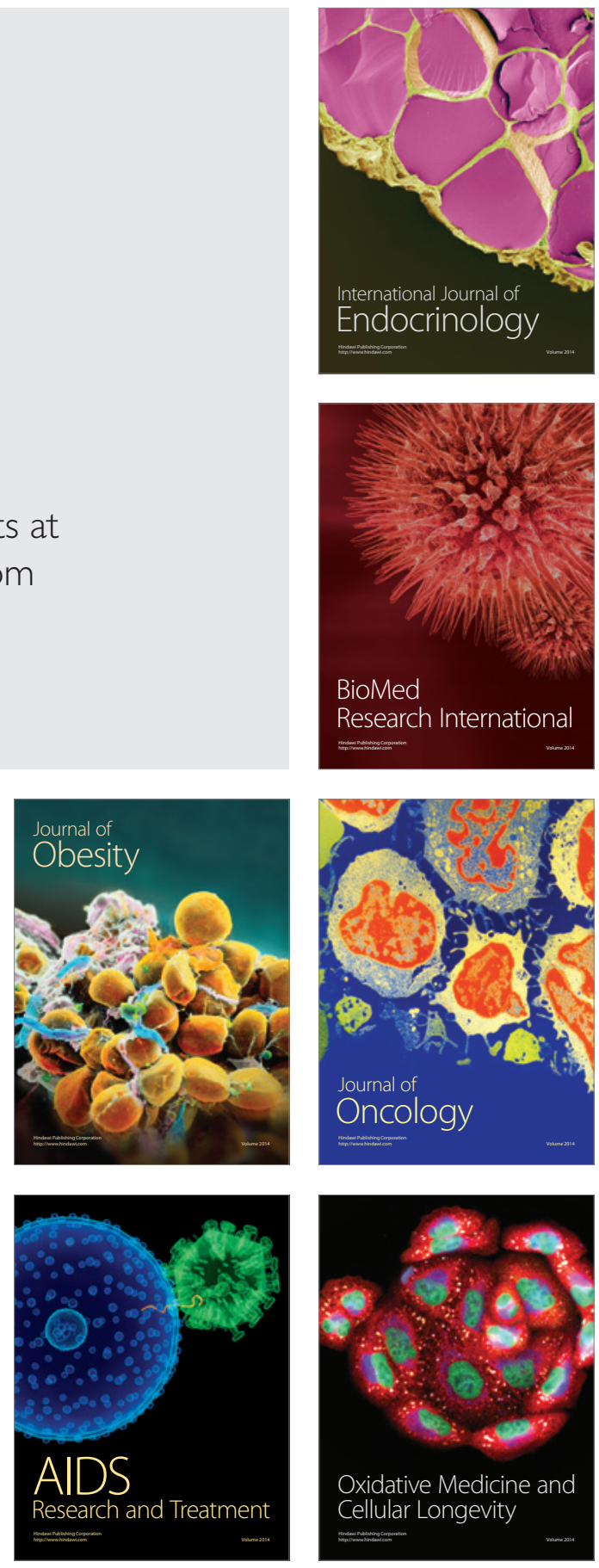